\title{
MULTIMODE CALCULATION OF FREQUENCY TUNABLE GYROTRONS FOR DYNAMIC NUCLEAR POLARIZATION APPLICATIONS
}

\author{
Liu Yinghui ${ }^{1,2}$, Kern, Stefan ${ }^{2}$, Matthias H. Beringer ${ }^{2}$, \\ Manfred Thumm ${ }^{2,3}$ \\ ${ }^{1}$ Research Institute of High Energy Electronics, University of \\ Electronic Science and Technology of China, 610054 \\ Chengdu, China \\ ${ }^{2}$ Forschungszentrum Karlsruhe, IHM, D-76021 \\ Karlsruhe, Germany \\ ${ }^{3}$ Universität Karlsruhe, IHE, D-76131 Karlsruhe, Germany \\ Stefano Alberti, Jean-Philippe Hogge \\ Centre de Recherche en Physique des Plasmas, Association \\ EURATOM-Conféd., EPFL, CH-1015 Lausanne, Suisse
}

Multimode calculations of a low-power (above 10W) highfrequency $(200 \mathrm{GHz}-300 \mathrm{GHz}$ ) gyrotron for NMR (nuclear magnetic resonance) spectroscopy applications have been performed. Six transverse modes $\left(\mathrm{TE}_{-7,2} ; \mathrm{TE}_{7,2} ; \mathrm{TE}_{-4,3} ; \mathrm{TE}_{4}\right.$, $\left.{ }_{3} ; \mathrm{TE}_{-2,4} ; \mathrm{TE}_{2,4}\right)$ whose coupling factors are above $30 \%$ and most probably excited in the cavity were included into the calculation. The frequency fine tuning was obtained via the excitation of a sequence of longitudinal modes of $\mathrm{TE}_{-7,2, \mathrm{q}}$ by varying the beam voltage from $15 \mathrm{kV}$ upward and the magnetic field from 9.6T to 9.77T. The diffractive quality factor of the cavity equals to 9965 and the ohmic quality factor of the cavity equals to 6414 . The results show that the main mode $\mathrm{TE}_{-7,2}$ is quite stable against the possible transverse mode competitors within this magnetic field range and a continuous frequency tuning range more than $800 \mathrm{MHz}$ $(263.43 \mathrm{GHz}-264.28 \mathrm{GHz})$ has been achieved with alpha equaling 1.3 , the beam radius and current being 1.33 and $100 \mathrm{~mA}$ respectively.

1. K. Kreischer, C. Farrar, R. Griffin, R. Temkin, and I. Vieregg, "A $250 \mathrm{GHz}$ Gyrotron for NMR Spectroscopy", 27th International Conference on Plasma Sciences, June 4-7, 2000, pp. 198.

2. Hornstein, M.K.; Bajaj, V.S.; Griffin, R.G.; Kreischer, K.E.; Mastovsky, I.; Shapiro, M.A.; Sirigiri, J.R.; Temkin, R.J., "Second harmonic operation at $460 \mathrm{GHz}$ and broadband continuous frequency tuning of a gyrotron oscillator", IEEE Trans. Electron Dev., vol. 52, no. 5, pp. 798-807, May. 2005. 3. La Agusu,; Murase, H.; Idehara, T.; Saito, T.; Mitsudo, S.; Takahashi, D.; Fujiwara, T., "Design of a $400 \mathrm{GHz}$ Gyrotron for DNP-NMR Spectroscopy", 31th International Conference on Infrared Millimeter Waves and 14th International Conference on Terahertz Electronics, Sept 18-22, 2006, pp. 82. 